\title{
Fourier domain excision of periodic radio frequency interference
}

\author{
Yogesh Maan ${ }^{1}$, Joeri van Leeuwen ${ }^{1,2}$, and Dany Vohl ${ }^{1}$ \\ 1 ASTRON, The Netherlands Institute for Radio Astronomy, Postbus 2, 7990 AA Dwingeloo, The Netherlands \\ e-mail: maan@astron.nl \\ 2 Anton Pannekoek Institute for Astronomy, University of Amsterdam, Science Park 904, 1098 XH Amsterdam, The Netherlands \\ Received 18 December 2020 / Accepted 22 March 2021
}

\begin{abstract}
The discovery and study of pulsars and fast radio bursts (FRBs) in time-domain radio data is often hampered by radio frequency interference (RFI). Some of this terrestrial RFI is impulsive and bright, and relatively easy to identify and remove. Other anthropogenic signals, however, are weaker yet periodic, and their persistence can drown out astrophysical signals. Here we show that Fourierdomain excision of periodic RFI is an effective and powerful step in detecting weak cosmic signals. We find that applying the method significantly increases the signal-to-noise ratio of transient and periodic pulsar signals. In live studies we detected single pulses from pulsars and FRBs that would otherwise have remained buried in background noise. We show the method has no negative effects on pulsar pulse shape, and that it enhances timing campaigns. We demonstrate the method on real-life data from a number of large radio telescopes, and conclude that Fourier-domain RFI excision increases the effective sensitivity to astrophysical sources by a significant fraction, which can be even larger than an order of magnitude in the case of strong RFI. An accelerated implementation of the method runs on standard time-domain radio data formats and is publicly available.
\end{abstract}

Key words. methods: data analysis - methods: observational - techniques: miscellaneous - pulsars: general - stars: magnetars stars: neutron

\section{Introduction}

As both the aperture area and the bandwidth of radio telescopes increase, the sensitivity with which the radio sky can be probed continues to increase. However, the potentially achievable sensitivity is often severely limited by the ever-increasing radio frequency interference (RFI). Preventive measures, such as using remote RFI-free locations to construct the new telescopes, identifying and isolating the sources of RFI (Anterrieu et al. 2016), and avoiding the known RFI-prone parts of the spectrum (e.g., Maan et al. 2013), are crucial for obtaining useful data from radio telescopes. However, even after exploiting these and other preventive methods, data obtained from radio telescopes are often significantly contaminated by RFI. Much of the radio spectrum used by new broadband receivers is outside the protected spectrum. The quickly growing number of radio-emitting satellites affect telescopes even in radio-quiet and remote zones. The efficient mitigation of such RFI remains a challenging process.

The RFI mitigation methods are broadly divided in two categories: pre-detection and post-detection strategies. The predetection techniques often use reference signals or relatively simple statistical criteria to identify and mitigate RFI signals in real time (e.g., Baan et al. 2004; Buch et al. 2016). These techniques are often more suitable for highly impulsive or narrowband RFI. The hard requirement of real-time baseband processing might also pose implementation challenges, especially for wide-band systems and existing backends. The postdetection techniques mostly involve off-line processing, though some rare but useful examples of real-time excision also exist (e.g., Sclocco et al. 2019). These strategies often use thorough statistical techniques or exploit the expected signatures, either of the signal-of-interest or that of the RFI signal, to identify and mitigate RFI. There is no unique technique that can be used to mitigate all kinds of RFI, given the ever-growing number of RFI sources and the many different ways in which they manifest themselves in the radio astronomical data. On the one hand, mitigation techniques include identifying outliers using first- and second-order moments or other simple Gaussian statistics (e.g., Baddi 2011), or using higher-order statistics (Dwyer 1983) such as spectral kurtosis (Nita et al. 2007; Nita \& Gary 2010). On the other hand, methods that focus on specific types of astronomical (e.g., Eatough et al. 2009; Hogden et al. 2012) or RFI signals are successful while a robust statistics approach might fail (e.g., Deshpande 2005; Lazarus et al. 2020).

Many robust statistical approaches have been developed to excise primarily the RFI instances with spiky or burst-like characteristics. These techniques are not readily applicable when the data are heavily affected by periodic RFI. One well-known source of periodic interference is the overhead 50 or $60 \mathrm{~Hz}$ powerlines that can produce radio noise by partial discharge at insulators and the sharp points of conductors. Moreover, most household and commercial electrical appliances also operate on 50 or $60 \mathrm{~Hz}$ alternating current. If not properly designed or shielded, these appliances might generate interference with the corresponding 50 or $60 \mathrm{~Hz}$ periodicity. Several other sources, such as military, aviation, and airport radar systems, or even ionosphere-research radar could also cause periodic interference, though the periods of such interference are hard to know in advance. Electric fences also cause interference with periods of nearly one second, but the actual period could differ depending on the design. Windmills also cause periodic interference, though the periods could be as short as a few microseconds. The periods of interference from these and many unknown sources could also vary significantly over time.

Contamination by periodic interference changes the apparent nature of the underlying statistical distribution, making it 

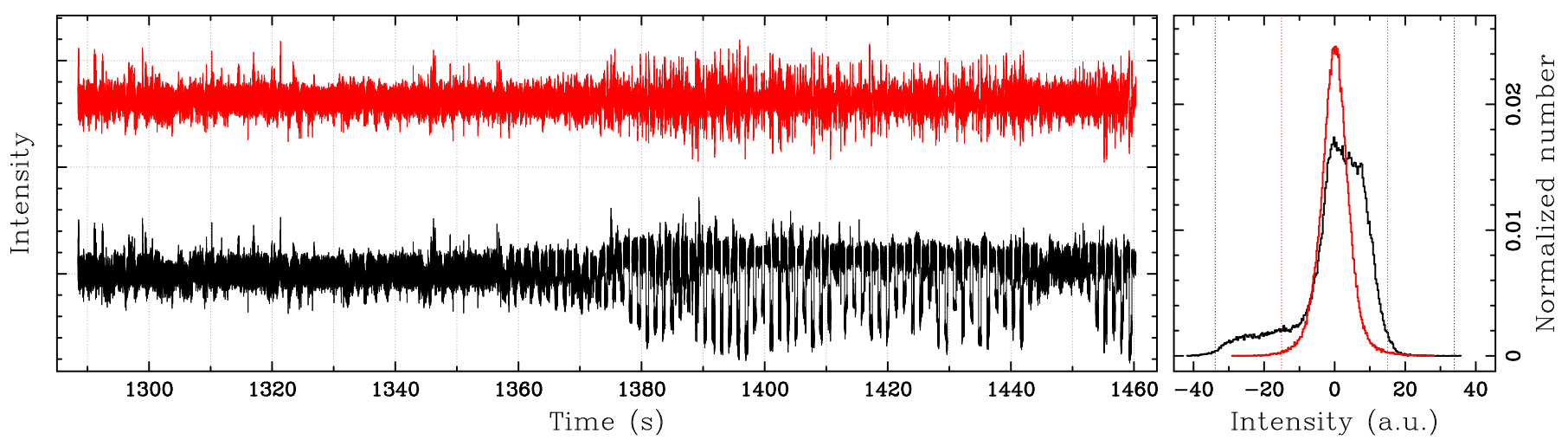

Fig. 1. A demonstration of Fourier domain excision of periodic RFI. Left panel: two intensity time sequences: the original sequence recorded at a radio telescope (black), the second half of which is evidently contaminated by periodic RFI, and the resultant sequence (red) when the strong frequency components in the Fourier transform of the original one are zapped. Both of the sequences were detrended for any slow variations before plotting. Right panel: normalized histograms for the two sequences shown on the left and the $\pm 3.5 \sigma$ bounds around the mean level marked as vertical lines, in the corresponding colors.

harder to identify RFI using conventional techniques. Moreover, in the case of such contaminations the excision tools that identify the strong periodic interference, such as rfifind from the pulsar search and analysis software PRESTO (Ransom et al. 2002), could potentially result in masking a large fraction of data. In the case of periodic RFI it is possible to largely remove the contribution of the periodic interference from the data, and hence avoid masking a large fraction as RFI-contaminated. In this work we describe a technique and present software tools to excise periodic interference in the Fourier domain, restore the underlying statistics, and then use the conventional methods for further RFI mitigation. We demonstrate the efficacy of this approach using real-life observations of primarily two types of astronomical sources, radio pulsars, and fast radio bursts (FRBs). Pulsars are fast-rotating neutron stars (Hewish et al. 1968), and the radio emission from them is observed in the form of a highly periodic sequence of regular pulses. FRBs are milliseconds-wide highly luminous radio transient events, most likely of extra-galactic origin (Lorimer et al. 2007; Thornton et al. 2013). While the presented technique and software are readily applicable to pulsar and FRB data, the underlying approach can be applied to timedomain radio data on any other astronomical source.

In what follows we elaborate on the impact of periodic RFI and its mitigation using the 1D Fourier transform in Sect. 2. In Sect. 3 we present details of a software tool RFIClean that we have developed to excise periodic and other RFI from timedomain data in the widely used filterbank format. We then briefly discuss mitigating wide-band, fainter periodic interference using the 2D Fourier transform and other future work in Sect. 4, followed by our conclusions in Sect. 5 .

\section{Periodic RFI: Impact and mitigation}

For a time sequence of radio intensity or power data, $f(t)$, the impulsive RFI-contaminated samples generally lie significantly outside the expected distribution of the non-contaminated samples. With averaging taking place at several stages in the datarecording systems, the distribution of the final time sequence is often very close to a Gaussian function. However, the presence of strong periodic RFI can significantly change the underlying Gaussian statistics.

Most RFI excision techniques rely strongly on the first two moments of the underlying distribution, the mean $(\mu)$ and standard deviation $(\sigma)$, to identify outliers. For a given threshold, $n$, the samples outside $\mu \pm n \sigma$ are treated as outliers. When the distribution is affected by periodic RFI, measurements of $\mu$ and $\sigma$ from the data themselves are likely to deviate significantly from their true values. In particular, $\sigma$ is likely to be overestimated, which could make the threshold-based identification of outliers naturally less efficient. For the example shown in Fig. 1, the $\mu \pm 3.5 \sigma$ bounds marked in the right panel for the original data demonstrate how ineffective they would be in identifying any outliers. By using more robust methods (e.g., the iterative methods), computing statistics using only RFI-free parts of the data, or using a reference signal the values of $\mu$ and $\sigma$ could be better estimated in some cases. However, these better estimates would then imply identification of significant fractions of data as outliers due to periodic RFI. For the example shown in Fig. 1, more than $10 \%$ of the data would be rejected as outliers above $n=3.5$. Moreover, the remaining fractions of data that are still contaminated by periodic RFI but not identified as outliers would leave their imprints in any further analysis of such data.

The Fourier transform decomposes the input function into a sum of complex exponentials (i.e., complex sinusoids) of specific frequencies. The amplitudes of the exponentials or the sinusoids would represent the amount of power in the input function at the corresponding frequency. For a discretely, uniformly sampled function $f(t)$ of $N_{t}$ samples, such as the time sequence shown in Fig. 1, the discrete Fourier transform (DFT) can be written as

$F\left(v_{t}\right)=\sum_{k=0}^{N_{t}-1} f(k \Delta t) e^{-2 \pi i\left(v_{t} k \Delta t\right)}$,

where $\Delta t$ is the sampling interval and $v_{t}$ is the Fourier frequency. For a real-valued function, $F\left(v_{t}\right)=F\left(-v_{t}\right)^{*}$. The inverse DFT, which would transform $F\left(v_{t}\right)$ back to $f(t)$, is of similar form but with a normalization constant, $1 / N_{t}$, and positive sign of the exponent. For a signal or RFI that modulates sinusoidally with a period $P_{s}$, the entire modulation power is concentrated at $v_{t}=1 / P_{s}$ in the DFT. If the modulation envelope differs from sinusoid, the modulation power is distributed between the fundamental $v_{t}=1 / P_{s}$ and its harmonics. When the modulation period varies with time, the modulation powers is distributed in more than one Fourier frequency bin around the fundamental and its harmonics in the DFT. A similar effect is seen when the input function length is a non-integer multiple of the modulation period (see, e.g., Ransom et al. 2002) In any case, power of a 
periodically modulated signal is concentrated within a very limited number of bins in the DFT, much fewer than in the time sequence $^{1}$. Hence, it is much easier to identify periodic signals in the Fourier domain.

For a time sequence of Gaussian distributed white noise, the real and imaginary parts of the Fourier transform also have Gaussian distributions with 0 mean and standard deviations equal to that of the input sequence. Since all the power of any periodic RFI in time-domain will be contained within far fewer frequency bins in the Fourier domain, it is much easier to identify the corresponding samples in the DFT. If these identified outliers are replaced by the expected mean of 0 in the real and imaginary parts of the DFT, an inverse DFT would then provide a time sequence wherein the periodic RFI is effectively mitigated. An example of this mitigation is shown in Fig. 1, where the periodic RFI apparent in the second half of the input sequence is successfully mitigated from the output sequence. We note that the Fourier-domain mitigation of periodic RFI does not lead to any loss of data samples in the time-domain. Even in the Fourier domain, for the above example, only $0.6 \%$ of the Fourier frequency space was blanked. A more readily evident effect is that the underlying distribution is restored (right panel), making the time sequence much more suitable for conventional RFI excision. For the same thresholds, only $0.8 \%$ of samples in the Fourier domain-cleaned time sequence are found to be outliers, as opposed to more than $10 \%$ in the input sequence. More importantly, restoration of the underlying distribution helps to identify actual impulsive RFI samples which could have otherwise been hindered by the periodic RFI modulation in the input sequence.

\section{Mitigating periodic and other RFI from time-domain data: RFIClean}

The method for mitigating periodic RFI described above has been successfully used in pulsar searches and studies (e.g., Faulkner et al. 2004; Smits et al. 2005; Camilo et al. 2007). However, the usage has generally remained limited to bandwidth-averaged time series, and for RFI with already known periodicities. As the strength, and possibly even phase, of the periodic interference could be highly variable across the observing bandwidth, its identification and mitigation might not always be efficient in the bandwidth-averaged time series. Moreover, mitigating some periodicities that are frequently caused by known RFI (e.g., $50 \mathrm{~Hz}$ ) in the band-averaged time series might also cause astronomical signals with unfavorably close fundamental or (sub-)harmonics to be lost. This was potentially the cause of the initial non-detection of PSR J2030+3641 by Barr et al. (2013). Furthermore, with the ever-growing number of RFI sources, it is not always possible to know the RFI periodicity in advance. With these considerations we present below an approach to mitigate periodic RFI (1) before averaging over the bandwidth, (2) only when the RFI is strong enough to be detectable, and (3) without a priori information on its periodicity.

A widely used format for time-domain data recorded at radio telescopes is the filterbank format: uniformly time-sampled streams at a number of frequency channels across the observing bandwidth. In this section we describe a software package, RFIClean ${ }^{2}$, which we developed to identify and mitigate periodic and impulsive RFI from filterbank data. The methods used

\footnotetext{
1 An exception is when the modulating signal approaches the Dirac comb function.

2 https://github.com/ymaan4/rficlean
}

are general purpose in the sense that no a priori information about the periodicity or source of RFI is needed. In the following subsections we provide an operational overview of the package followed by details on its computational and scientific performance using real data.

\subsection{Operational overview and diagnostics}

RFIClean reads in and works on the input data block by block, where a block represents time series of a user-specified length for a number of radio frequency channels. Three categories of RFI excision are employed. The first of these categories is the Fourier domain excision. For each time series a DFT is computed using the Fastest Fourier Transform in the West (FFTW; Frigo \& Johnson 2005) software library. The Fourier amplitude spectrum is often red (i.e., there is more spectral power at lower frequencies than that at higher frequencies), which prohibits efficient identification of any outliers. To avoid the worsening effects of this redness, the amplitude spectrum is divided into several sections; the length of each section is increased logarithmically as we go from lower to higher Fourier frequencies. The outliers are identified within each of these sections using robustly computed means and standard deviations, and a specified threshold. For each of the outliers, the real and imaginary parts of the DFT are replaced by $0 \mathrm{~s}$. An inverse DFT computed using FFTW then outputs a cleaned time series. This operation is repeated for time series corresponding to each of the radio frequency channels in the input block.

Next, narrowband RFI is identified and excised from the above DFT-cleaned block. For this to happen the mean and variance spectra are obtained for the block by computing a robust mean and standard deviation of each of the time series. The channels that show either the mean or the variance power above a specified threshold are identified and flagged as outliers. The spectral power distribution in data obtained from radio telescopes is often significantly affected by electronic filters and other parts in the backends. This might make the above threshold-based identification of contaminated channels in the mean and variance spectra less effective. To overcome any instrument-induced spectral variations we compute the differences between the mean spectrum values at adjacent channels; the resultant spectrum is known as the difference-mean spectrum. Hence, using the mean spectrum $\left(S_{\text {mean }}\right)$ the differencemean spectrum $\left(\Delta S_{\text {mean }}\right)$ is computed as

$\Delta S_{\text {mean }}(j)=\sum_{j=0}^{N_{f}-2}\left[S_{\text {mean }}(j)-S_{\text {mean }}(j+1)\right]$,

where $N_{f}$ is the number of radio frequency channels. We note that $\Delta S_{\text {mean }}$ consists of $N_{f}-1$ elements. With a similar approach we use the variance spectrum to compute the difference-variance spectrum. These difference-spectra, nearly free from the effects of the spectral shapes induced by the instrument, are then used to further identify the outlier channels. The time series in every identified outlier channel is replaced by the mean of the adjacent non-contaminated channel. The spectra for the individual time samples are also examined to find any narrowband and narrowtime RFI, and the identified outliers are replaced by the specified threshold.

In the last excision step the time series computed by averaging the above DFT-and-spectral-cleaned block over all the channels is then subjected to threshold-based excision. This step identifies any impulse-like RFI, and replaces the corresponding 


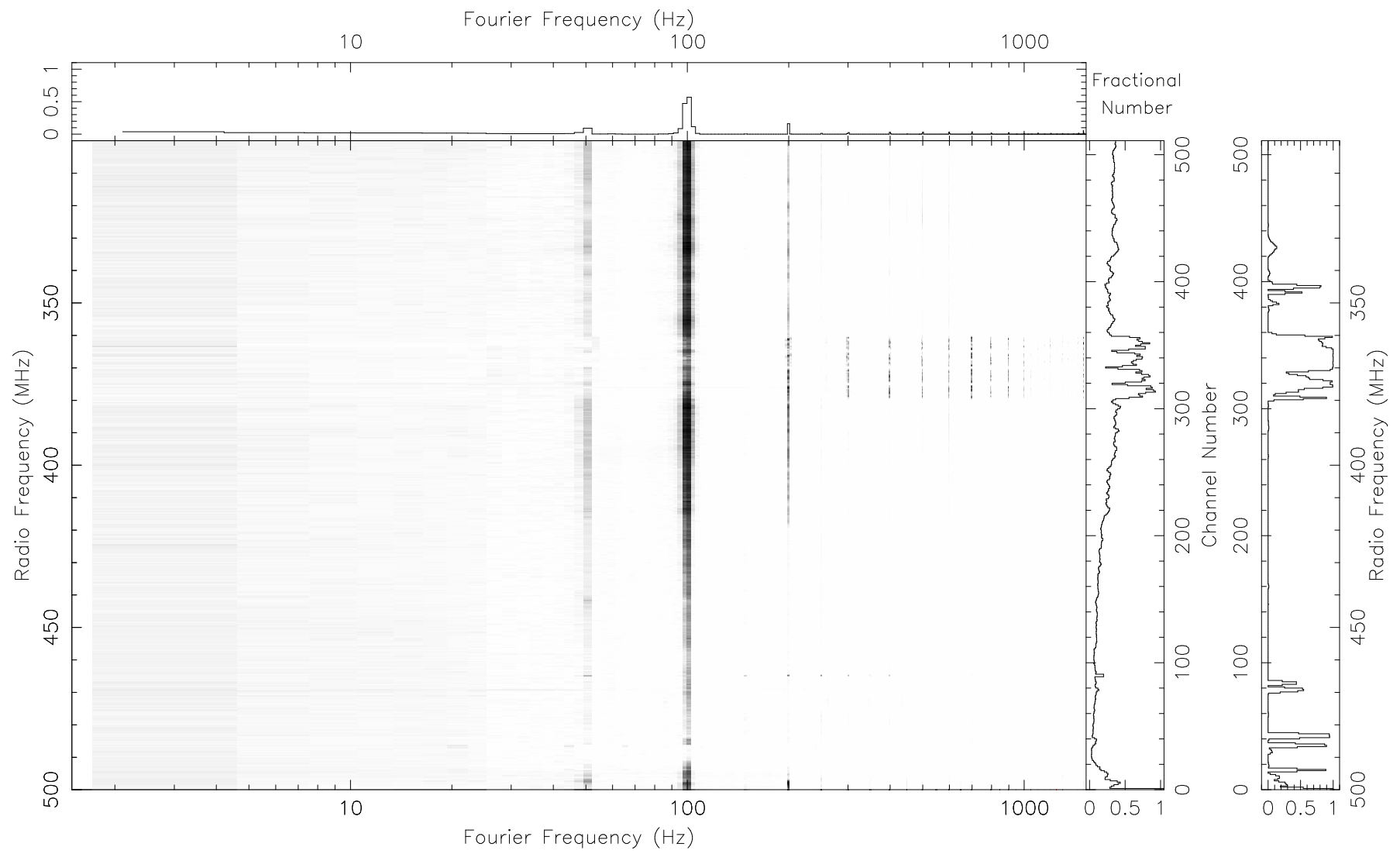

Fig. 2. Diagnostic plot output from RFIClean. The composite plot on the left shows the statistics of the Fourier frequencies that were excised. For every radio frequency channel in the main panel, the grayscale mapping shows how often a Fourier frequency was excised. Darker colors indicate larger numbers. The adjacent panels at the top and on the right show the averages along the vertical and horizontal directions, respectively. The far right panel shows how often the radio frequency channels were identified as narrowband RFI and excised (see text for more details).

samples for each of the frequency channels by means of their corresponding time series.

A diagnostic is output at the end that primarily indicates the fractional number of times a Fourier frequency was zapped for each of the radio frequency channels, and how many times a channel was zapped in the spectral cleaning. An example of a diagnostic plot using time-domain data obtained from the Giant Metrewave Radio Telescope (GMRT) is shown in Fig. 2. As is evident from the main panel, periodic interference at around $50 \mathrm{~Hz}$ and its first two harmonics $(100$ and $150 \mathrm{~Hz})$ were zapped the most. The top panel suggests that the $100 \mathrm{~Hz}$ interference was identified and zapped from the time series for all the radio frequency channels, on average, about $50 \%$ of the times. Corresponding to this interference, the zapped frequencies are spread over 4-5 Fourier bins, indicating the jitter in period of the interference. More than ten harmonics of $50 \mathrm{~Hz}$ are prominently visible particularly in the frequency range $360-380 \mathrm{MHz}$, a part of the spectrum known to be often dominated by interference at GMRT. As apparent from the far right panel in Fig. 2, the spectral zapping block identified and excised this fairly broad RFI (about $20 \mathrm{MHz}$ ) as well as several narrowband RFIs.

\subsection{Computational performance}

Although RFIClean was primarily developed for excision of RFI in the offline processing of data, the execution time could be of importance for processing large data sets or if real-time excision of periodic RFI is desired. To utilize more than one processing core, RFIClean offers two methods of parallel processing.
In the first method, a given filterbank data set is virtually divided into a number $n$ of time-segments, and individual serial instances of RFIClean are used to process these segments simultaneously and write the cleaned data in separate output files. At the end, the output files are concatenated in the correct order to output a single RFI-excised filterbank file. A bash script is provided along with RFIClean, which facilitates the above parallel processing for a specified number of processing cores.

For the second method we first identified the most computeintensive functions within RFIClean using the GNU profiler ${ }^{3}$. In the serial form of some of these functions, frequency channels are processed independently from one another. Hence, it represents a case of a "pleasingly parallel problem." We modified data structures and parallelized sections of algorithms employing the OpenMP architecture ${ }^{4}$, such that channels can be processed and cleaned in parallel blocks over $n$ threads.

We benchmarked the execution time using five filterbank files parallelized over $n \in\{1,2,4,8,16\}$ processing units, where a processing unit corresponds to the number of processes used in the bash-script method, and the number of threads for the OpenMP method. For this benchmarking, we used filterbank data with observing durations of about 5, 11, 22, 43, and 87 minutes, each with 2048 frequency channels and $163.8 \mu$ s sampling time. RFIClean was specified to use time blocks with 8192 samples to identify and excise RFI on a computing machine with

\footnotetext{
3 https://sourceware.org/binutils/docs/gprof/, last visited 11 November 2020.

4 https: //WWW . openmp. org, last visited 11 November 2020.
} 


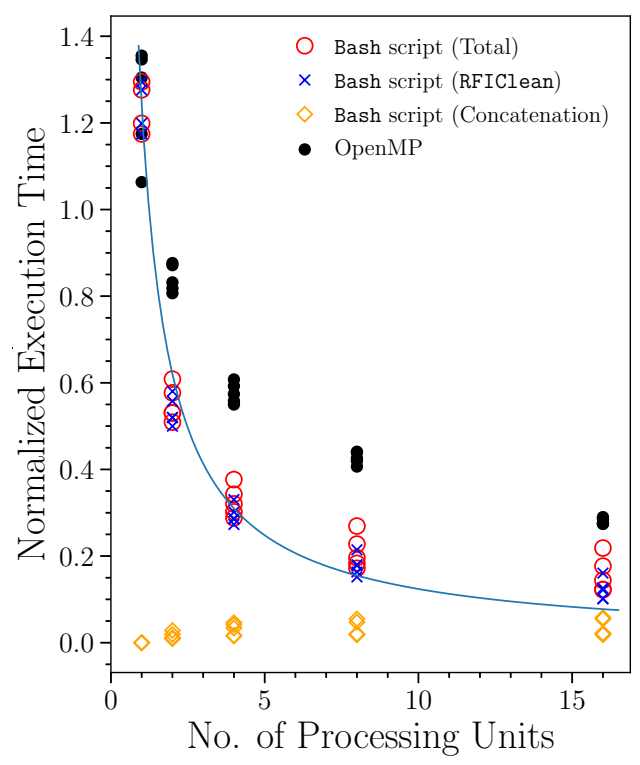

Fig. 3. Processing time spent per one second of observing duration as a function of number of processing units are shown for two parallelization methods. In the bash-script method, the time spent by the multiple RFIClean instances, for concatenating the partial data products, and the total time are shown separately using the red, blue and orange symbols. The time spent using the OpenMP method is shown in black. The blue curve shows the theoretical gain offered by an $n$-unit parallelization which splits perfectly the workload, based on the average normalized execution time obtained from the serial bash-script method (i.e., for a single processing unit; 1.24 seconds/second).

24 CPUs (2 Intel(R) Xeon(R) E5-2620v3 processors), $125 \mathrm{GiB}$ memory, and CentOS Linux 7 operating system. We normalized the benchmarked execution times by the observing durations.

Results of the benchmark are shown in Fig. 3. For the first method, the execution time scales down close to the expected linear relationship (solid line) with the increasing number of processing cores. While using a large number of cores, the speed-up in this method is primarily limited by disk I/O. The fractional time spent in concatenating the simultaneously processed data into a single output also becomes significant while using large number of cores. For the above-mentioned sampling time and number of radio frequency channels, RFIClean can excise periodic and other RFI in real time using just two processing cores. As the Fourier transform computation time scales as $\Omega(N \log$ $N$ ), the execution time in Fig. 3 will also scale accordingly when a different block size is used. Similarly for the second method, RFIClean operates in real time starting as early as $n=2$. The execution time $T$ scales with the number of threads, providing speed-ups $\left(T_{\text {serial }} / T_{n}\right)$ of about 5 when using 16 threads. As can be seen, this method is slightly slower than the first method. This is primarily due to the fact that operations related to input read and output write, and some of the functions that are not as compute-intensive, are performed serially in the present version. On the other hand, this method has the advantage of simplicity of execution and setup while providing good acceleration.

\subsection{Scientific performance}

Here we demonstrate how using RFIClean could improve the fraction of data that is lost in conventional RFI excision, and its efficacy in bringing up faint astronomical signals (particularly in pulsar and FRB searches). We also show that RFIClean can

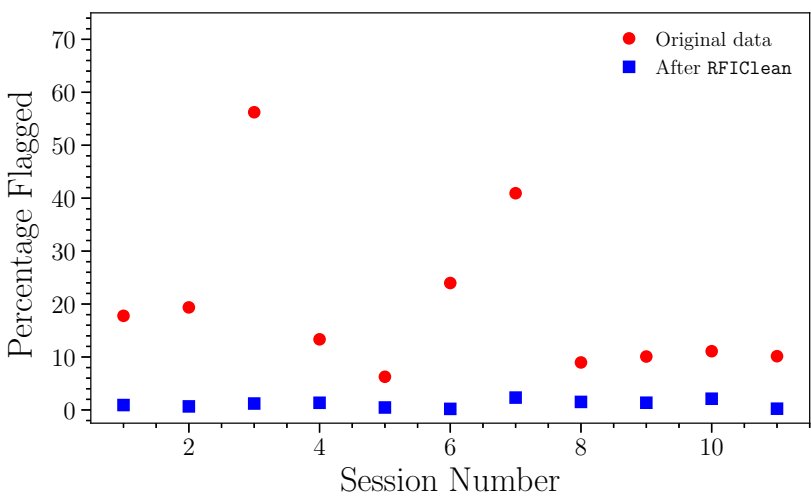

Fig. 4. Percentage of data masked by rfifind before and after using RFIClean for a number of observing sessions. A significant improvement in the percentage of unmasked data is clearly evident.
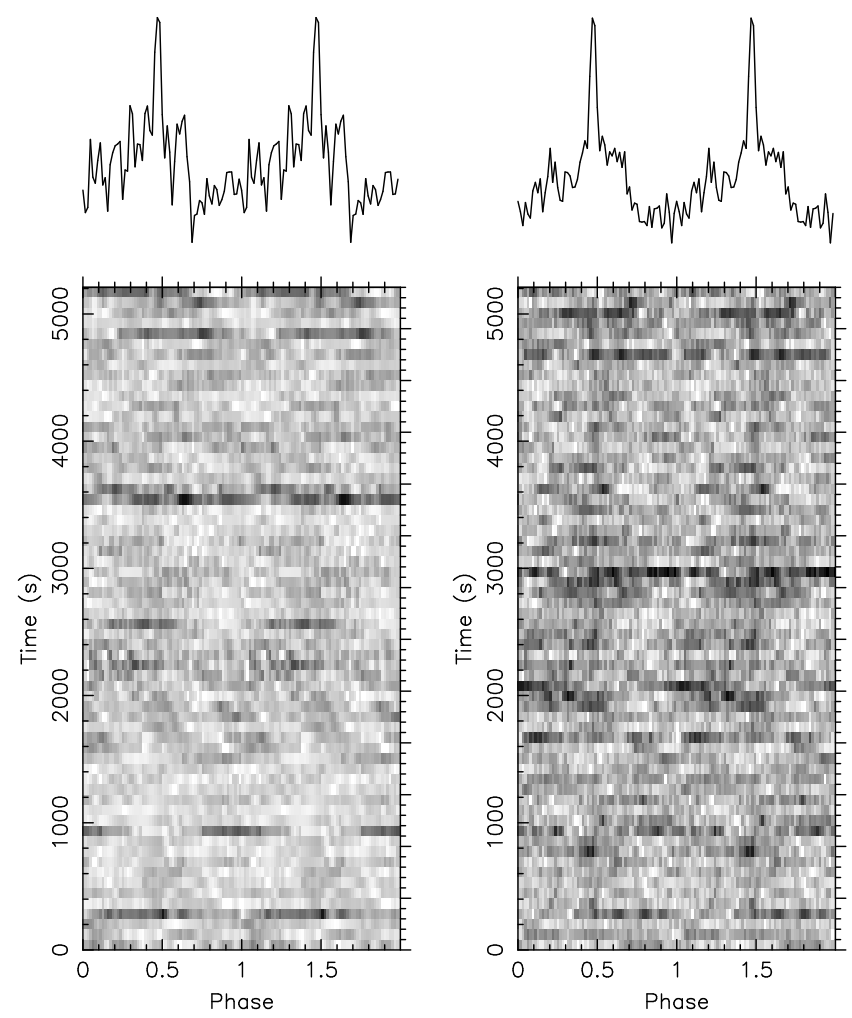

Fig. 5. Detection of periodic signal from the pulsar J0533-4524 (Oostrum et al. 2020) before (left) and after (right) using RFIClean. The lower panels show a stack of partial average profiles obtained by folding over the pulsar's period, with each row corresponding to about $80 \mathrm{~s}$ of data, and the darker colors indicate higher intensities. The upper panels display the vertical averages of data displayed in the lower panels, and correspond to the net average profile of the pulsar. Improvement in the detection significance of the pulsed periodic emission is clearly visible.

safeguard the periodic signals from bright pulsars while mitigating periodic RFI, does not introduce any artifacts on the pulse profiles obtained from cleaned data, and improves the quality of the precision timing experiments.

For some of the demonstrations below we used observations of a very bright pulsar, J0437-4715, conducted using GMRT on a number of epochs between November 3, 2018, and March 1, 2019. In each observing session, data were recorded 

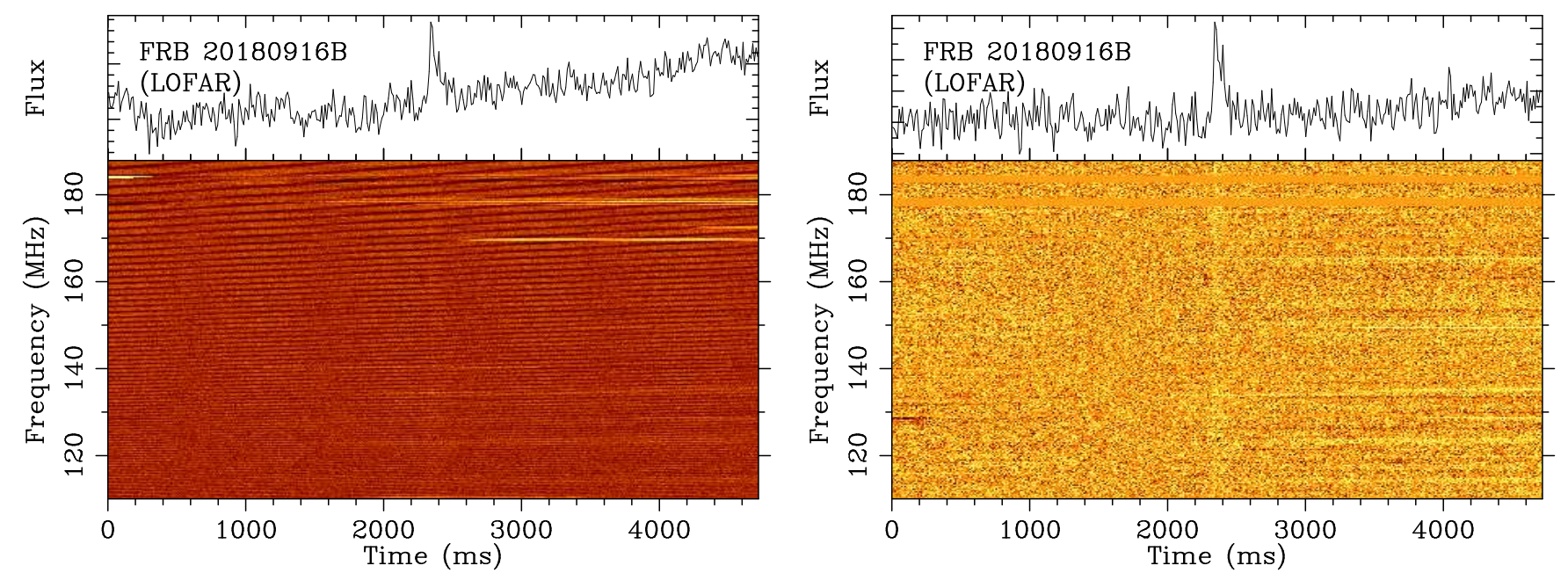

Fig. 6. Detection of a faint burst from the repeating FRB 20180916B at $150 \mathrm{MHz}$ before (left) and after (right) using RFIClean. The lower panels show the spectrograms around the burst arrival time, after correcting for the known dispersive delay across the bandwidth. Due to the dispersive delay corrections, the periodic RFI appears as highly slanted stripes in the left image. The upper panels display the vertical averages of data displayed in the lower panels, and correspond to the net average profile of the burst. Improvement in the detection confidence and overall data quality of the burst is clearly visible.

with 2048 frequency channels and $0.16384 \mathrm{~ms}$ sampling time, and the observing durations varied between 2 and 6 minutes.

\subsubsection{RFI excision efficiency}

To demonstrate RFIClean's efficacy in improving the fraction of available data that is not masked by conventional RFI excision methods, we used rfifind from PRESTO. The rfifind tool examines data for narrow- and broadband interference as well as strong periodic interference, produces a mask for the parts of the data that it finds RFI-contaminated, and reports the masked percentage of the data. For the above observations of J0437-4715, the percentage of data that are masked by rfifind before and after using RFIClean are shown in Fig. 4. A sharp improvement in the percentage of the data that rfifind finds RFI-free after mitigation using RFIClean is clearly evident for all the observing sessions.

\subsubsection{Usefulness in pulsar and FRB searches}

In pulsar and FRB searches the systematic noise due to RFI reduces the $\mathrm{S} / \mathrm{N}$ of the underlying astronomical signals, and increases the number of false candidates that a human or machine has to sift through before identifying the genuine ones. This also decreases the probability of any faint but genuine candidates being identified correctly with the required confidence. By efficient excision of periodic RFI, as well as narrow- and broadband RFI, RFIClean helps in uncovering the underlying faint signals.

Detection of the recently discovered pulsar J0533-4524 (Oostrum et al. 2020) at several epochs was in fact possible only after the mitigation of periodic RFI using RFIClean. An example of the improvement in the pulsar's S/N after using RFIClean on a GMRT observation of this pulsar in the $300-500 \mathrm{MHz}$ band is shown in Fig. 5, where the formal $\mathrm{S} / \mathrm{N}$ increased from less than 3 to about 25. Detection of the magnetar J1809-1943 below $500 \mathrm{MHz}$ (Maan et al. 2019) was also immensely aided by RFIClean. In radio searches where information about the pulsar period is already available (e.g., from high-energy counterparts, Maan \& Aswathappa 2014; Maan 2015), RFIClean can help in realizing high sensitivity by safeguarding the known periodicity (see Sect. 3.3.3).

More recently, a number of bursts from the repeating FRB 20180916B have been discovered at $150 \mathrm{MHz}$ using the Low Frequency Array (LOFAR) telescope (Pastor-Marazuela et al. 2020), marking the first convincing detection of any FRB at frequencies below $300 \mathrm{MHz}$. The first of these detected bursts (L01), which is also the faintest, was confirmed only after some strong periodic RFI was excised from the LOFAR data. A spectrogram around the burst arrival time obtained from the original data and that from data cleaned using RFIClean is shown in Fig. 6. In the process of correcting for the large dispersive delay (nearly $79 \mathrm{~s}$ ) across the observed bandwidth for FRB 20180916B, the originally undispersed periodic RFI actually became dispersed, and it appears in the form of highly slanted stripes in the spectrogram. As evident in Fig. 6, RFIClean successfully excised the periodic RFI as well as some narrowband RFI. In this particular case, due to the uniform amplitude of the periodic RFI across the bandwidth and to the very large dispersive delay that had to be corrected, the effect of the RFI on the formal S/N of the burst was only marginal. However, confirmation of the burst as an astronomical signal as well as study of its flux density and spectrum were possible only after the RFI was mitigated using RFIClean. The mitigation of periodic RFI from these data also reduced the false burst candidates by nearly an order of magnitude.

\subsubsection{Usefulness in pulsar timing experiments}

Pulsars are excellent celestial clocks. Given the stability of their periods up to one part in $10^{20}$, measuring the time of arrival (ToA) of their pulses facilitates very high precision astrophysical experiments such as testing the general relativity, constraining the equation of state, and detecting sub- $\mu \mathrm{Hz}$ gravitational waves.

Measuring the pulse ToA includes comparison with a template profile. Any small change in either the intrinsic pulseshape or that resulting from instrumental or processing software artifacts, would result in an offset and/or increased uncertainty in the corresponding ToA measurement. To avoid a possible 

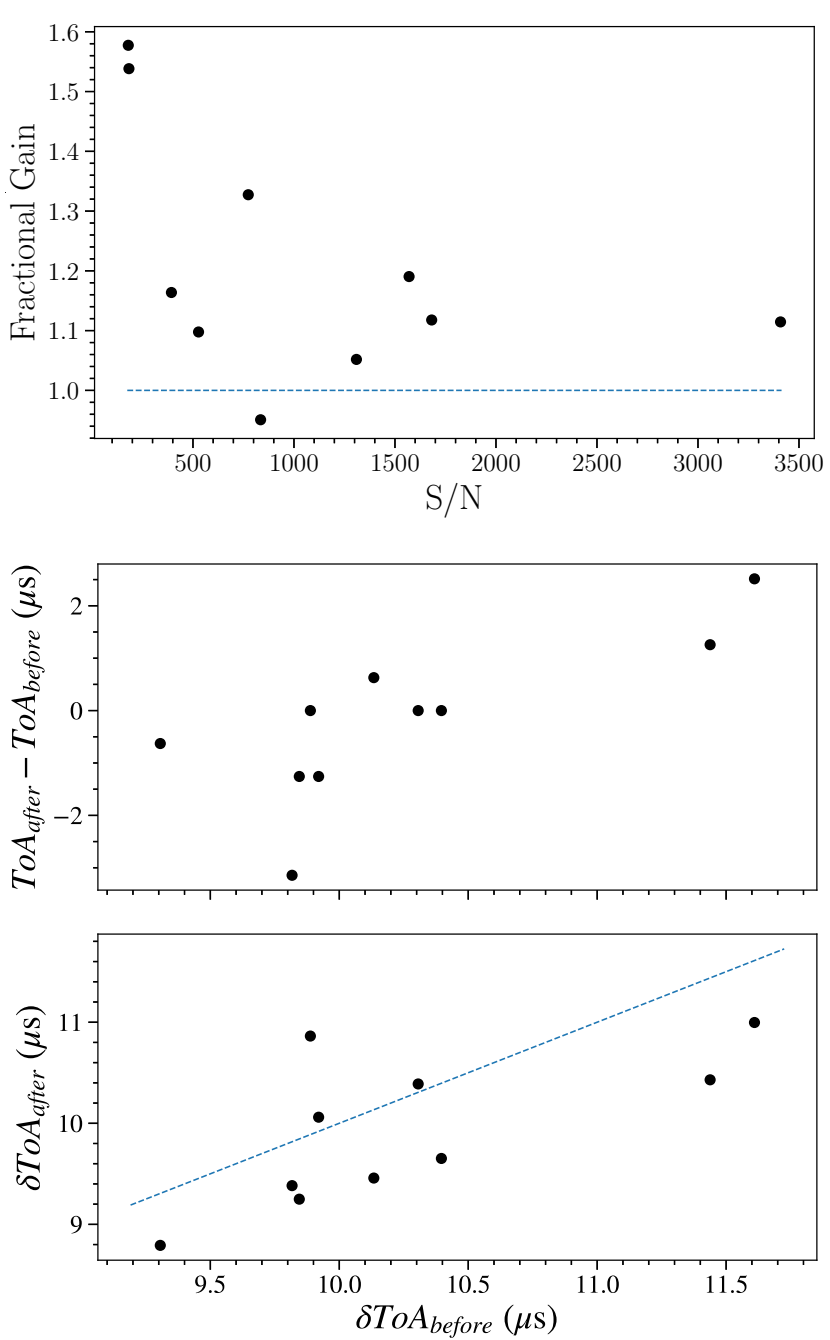

Fig. 7. RFIClean's suitability in timing studies: the upper panel shows the fractional increase in $\mathrm{S} / \mathrm{N}$ after excision using RFIClean, as a function of the $\mathrm{S} / \mathrm{N}$ obtained from the original data. The middle panel shows the difference in times-of-arrival (ToA) measured from the original and the RFI-excised data, as a function of the associated uncertainties in the original ToAs. The difference is much smaller than the associated uncertainties. The bottom panel shows a comparison between the ToA uncertainties measured before and after the RFI excision. The uncertainties in measurements obtained from the RFI-excised data are consistently smaller than their counterparts from the original data for most of the observations.

excision or modification of the periodic signal from strong pulsars, RFIClean can safeguard the Fourier frequencies corresponding to a pulsar's specified spin period.

We demonstrate RFIClean's suitability for high precision pulsar timing experiments using the observations of the strong pulsar J0437-4715 discussed earlier in this section. As can be seen in the top panel of Fig. 7, even without any RFI excision the pulsar's strong periodic signal is detected with high significance ( $S / N>200$, after averaging over bandwidth and observing duration) in all the observations. However, using RFIClean further improves the detection significance for most of the observations. The fractional improvement is naturally greater where the original detection significance was smaller. We also note that using rfifind on the data output from RFIClean further improves the $\mathrm{S} / \mathrm{N}$ by $10-20 \%$ in many cases (not shown in Fig. 7, but assessed separately).
To generate a template, we used psrsmooth from PSRCHIVE (Hotan et al. 2004) on the profile with the highest $\mathrm{S} / \mathrm{N}$. We then used pat on average profiles obtained from all the observing sessions to compare with the above template and measure the ToAs. The same template was used on the original data and on the RFI-excised data. As shown in the middle panel of Fig. 7, the ToAs obtained from the original and RFIexcised data are consistent with each other, and any difference is much smaller than the associated uncertainty. This indicates that RFIClean does not inflict any artifacts in the pulsar's profile shape. The lower panel shows that, even for this strong pulsar, the measurement uncertainties in the ToAs from the RFI-excised data are smaller than their counterparts from the original data for most of the sessions. The improvement in ToA uncertainty is a direct implication of the enhancement in $\mathrm{S} / \mathrm{N}$ shown in the upper panel. Much more significant improvements in ToA uncertainties have been observed for fainter pulsars, making RFIClean not only suitable, but also useful in improving ToA measurements in pulsar timing experiments.

RFIClean is an integral part of the data processing pipeline (Susobhanan et al. 2021) used in the Indian Pulsar Timing Array (InPTA) experiment. RFIClean is also being used in the pulsar timing studies at the Argentine Institute of Radio astronomy (Sosa Fiscella et al. 2021).

\section{Using two-dimensional DFT and other future work}

The identification and mitigation of periodic RFI in the previous section is limited by the strength of such RFI within individual channels. If a faint periodic RFI is spread over multiple channels or across the full observed bandwidth, the 2D discrete Fourier transform (2D DFT) might help in its identification and mitigation. While a detailed exploration in this regard will be the subject of future work, here we present a brief demonstration of mitigating periodic RFI using 2D DFT.

For a function discretely and uniformly sampled in two dimensions, $f(t, f)$, such as the filterbank data discussed in the previous section, the 2D DFT can be written as

$F\left(v_{t}, v_{f}\right)=\sum_{k=0}^{N_{t}-1} \sum_{j=0}^{N_{f}-1} f(k \Delta t, j \Delta f) e^{-2 \pi i\left(v_{t} k \Delta t+v_{f} j \Delta f\right)}$,

where $\Delta t$ and $\Delta f$ are the sampling intervals, $N_{t}$ and $N_{f}$ are the total number of samples, and $v_{t}$ and $v_{f}$ are the corresponding Fourier frequencies in the two dimensions. As for the 1D DFT, for a real-valued 2D function, $F\left(v_{t}, v_{f}\right)=F\left(-v_{t},-v_{f}\right)^{*}$. The inverse DFT, which would transform $F\left(v_{t}, v_{f}\right)$ back to $f(t, f)$, differs in the signs of the two exponents and a normalization constant, $1 /\left(N_{t} \times N_{f}\right)$.

For the demonstration, we used about $1.3 \mathrm{~s}$ of data from one of the GMRT observations on the bright pulsar J0437-4715 presented in the previous section. To make the interference visually apparent in the spectrograms, the already present interference by $50 \mathrm{~Hz}$ (and its harmonics) were manually enhanced by a factor of 4. The resultant spectrogram is shown in the top panel in Fig. 8, and the second panel shows its 2D DFT. Here $v_{t}$ is the Fourier frequency and $v_{f} \equiv$ Delay. Interference at $50 \mathrm{~Hz}$ and several of its harmonics are clearly visible. We note that the variation in the interference power along the vertical axis in this 2D DFT depends on how the interference power is distributed along various frequency channels in the spectrogram. In an ideal case, when the interference power is uniformly distributed across all the channels, it will show up only on the Delay $=0$ row in the 2D DFT. 

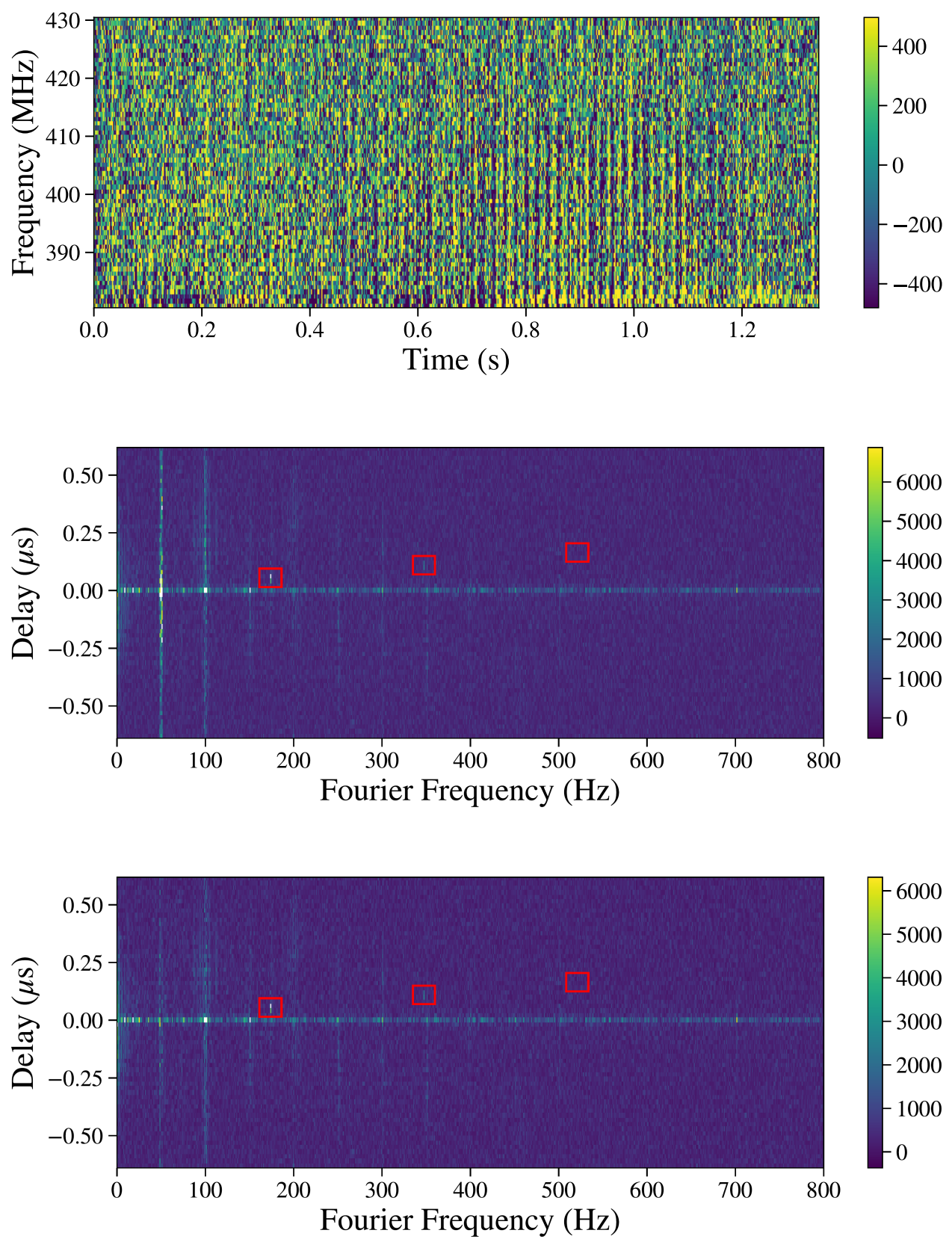

Fig. 8. A demonstration of deeper excision of periodic RFI using 2D DFT. Top panel: spectrogram of a $1.3 \mathrm{~s}$ of

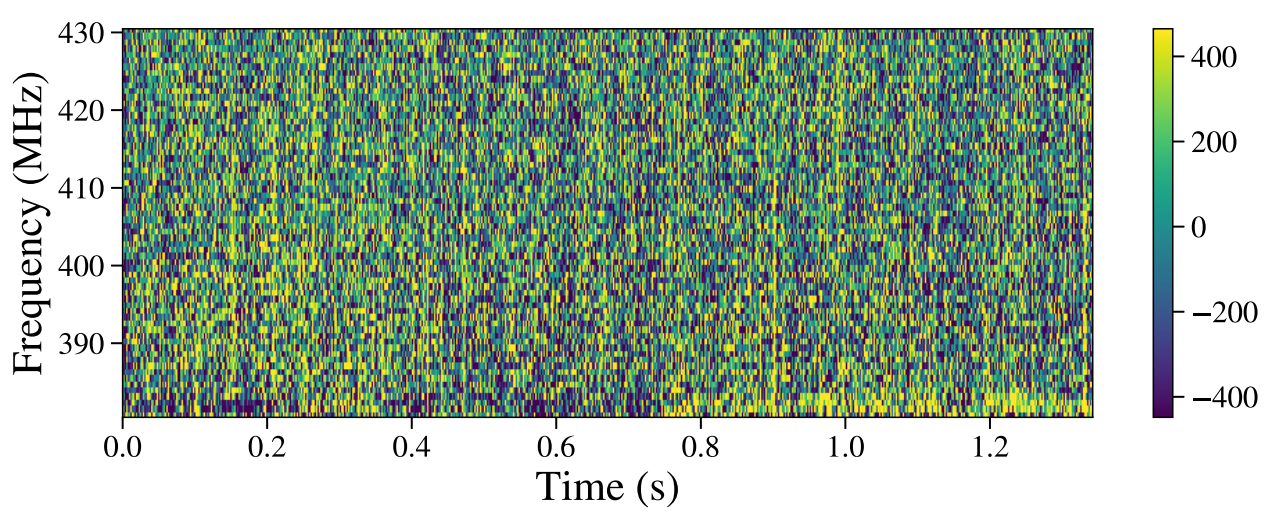
data from a GMRT observation of the bright pulsar J0437-4715, and with visible contamination by periodic $(50 \mathrm{~Hz})$ RFI. Second panel: 2D DFT of the data in the top panel. Third panel: 2D DFT after cleaning the data using RFIClean. Bottom panel: spectrogram after the periodic RFI are identified and excised using the 2D DFT. The red rectangles in the middle panels give the locations corresponding to the spin period of J0437-4715 and its first two harmonics, after taking into account the vertical offset caused by the inter-channel dispersive delays.

It should be noted that the periodic signal from the pulsar J0437-4715 is dispersed due to propagation effects in the ionized interstellar medium. As a consequence, the periodic train of pulses originated from the source exhibits a frequencydependent time delay. Due to this dispersed nature, the modulation power of periodic signal from the pulsar is expected to be 
offset from the Delay $=0$ row in the 2D DFT. An offset from the Delay $=0$ can also be used to distinguish between RFI and astronomical dispersed signals, which enables an efficient way of searching for new pulsars using 2D DFT. Camilo et al. (1996) used this method to search for millisecond pulsars, and more details of the method can be found therein. The expected locations corresponding to the pulsar's spin frequency and first two harmonics are indicated in the $2 \mathrm{D}$ DFT by red rectangles in Fig. 8. Even within this $1.3 \mathrm{~s}$ of data, the excess power due to the pulsar signal is clearly visible at the locations of the fundamental and first harmonic.

The third panel of Fig. 8 shows the 2D DFT after the spectrogram was cleaned using RFIClean. While a noticeable decrease in the interference power, primarily at $50 \mathrm{~Hz}$, is visible, it is evident that the RFI is not fully mitigated. To mitigate the remnant RFI, we computed the vertical average of the 2D DFT and identified the Fourier frequencies where the power exceeded a specified threshold. The data corresponding to these Fourier frequencies were replaced by local mean values in the 2D DFT. The cleaned spectrogram obtained by an inverse transform of the modified 2D DFT is shown in the bottom panel. While this simple approach efficiently mitigated most of the periodic RFI in this simple example, more complex contamination might require more sophisticated approaches.

New features for RFIClean are currently being planned, including reading and writing data in formats other than SIGPROC filterbank, and implementing the spectral kurtosis method (Nita \& Gary 2010), to mitigate non-periodic RFI even more efficiently. That said, the current version has already shown to be a powerful and sometimes essential tool in discovering and studying new pulsars and FRBs.

\section{Conclusions}

We presented a method to identify and mitigate periodic RFI in the Fourier domain, and showed that mitigating periodic RFI is essential for efficient identification and excision of other kinds of RFI. An accelerated software implementation of the method, RFIClean, is publicly available ${ }^{5}$. The method is shown to significantly enhance the effective sensitivity, and we presented a few examples where it has been instrumental in detecting faint pulsars and FRBs. Given its suitability and usefulness, the method is already being used in pulsar timing experiments. While our focus was to show the benefits on pulsar and FRB data, the method is directly applicable to a wide range of radio observations, particularly in the time-domain, of diverse astrophysical sources.

Acknowledgements. We thank our referee, Scott Ransom, for his comments and suggestions which have helped in improving the paper. D. V. utilized the Distributed ASCI Supercomputer 5 (DAS-5; Bal et al. 2016) during development and testing of the OpenMP parallelization of this software. The research leading to these results has received funding from the European Research Council under the European Union's Seventh Framework Programme (FP/2007-2013)/ERC
Grant Agreement n. 617199 ('ALERT'); from Vici research programme 'ARGO' with project number 639.043.815, financed by the Netherlands Organisation for Scientific Research (NWO); and from the Netherlands eScience Center (NLeSC) grant ASDI.15.406 ('AA-ALERT'). This paper is based (in part) on data obtained with the International LOFAR Telescope (ILT) under under project code COM_ALERT, OBSID L775795. LOFAR (van Haarlem et al. 2013) is the Low Frequency Array designed and constructed by ASTRON. It has observing, data processing, and data storage facilities in several countries, that are owned by various parties (each with their own funding sources), and that are collectively operated by the ILT foundation under a joint scientific policy. The ILT resources have benefitted from the following recent major funding sources: CNRS-INSU, Observatoire de Paris and Université d'Orléans, France; BMBF, MIWF-NRW, MPG, Germany; Science Foundation Ireland (SFI), Department of Business, Enterprise and Innovation (DBEI), Ireland; NWO, The Netherlands; The Science and Technology Facilities Council, UK; Ministry of Science and Higher Education, Poland.

\section{References}

Anterrieu, E., Khazaal, A., Cabot, F., \& Kerr, Y. 2016, Remote Sens. Environ., 180,76

Baan, W. A., Fridman, P. A., \& Millenaar, R. P. 2004, AJ, 128, 933

Baddi, R. 2011, AJ, 141, 190

Bal, H., Epema, D., de Laat, C., et al. 2016, Computer, 49, 54

Barr, E. D., Guillemot, L., Champion, D. J., et al. 2013, MNRAS, 429, 1633

Buch, K. D., Bhatporia, S., Gupta, Y., et al. 2016, J. Astron. Instrum., 5, 1641018

Camilo, F., Nice, D. J., Shrauner, J. A., \& Taylor, J. H. 1996, ApJ, 469, 819

Camilo, F., Ransom, S. M., Peñalver, J., et al. 2007, ApJ, 669, 561

Deshpande, A. A. 2005, Radio Science, 40, RS5S12

Dwyer, R. F. 1983, Proc. IEEE Int. Conf. on Acoustic, Speech, and Signal Processing (New York: IEEE), 8, 607

Eatough, R. P., Keane, E. F., \& Lyne, A. G. 2009, MNRAS, 395, 410

Faulkner, A. J., Stairs, I. H., Kramer, M., et al. 2004, MNRAS, 355, 147

Frigo, M., \& Johnson, S. G. 2005, Proc. IEEE, 93, 216, special issue on "Program Generation, Optimization, and Platform Adaptation"

Hewish, A., Bell, S. J., Pilkington, J. D. H., Scott, P. F., \& Collins, R. A. 1968, Nature, 217, 709

Hogden, J., Vander Wiel, S., Bower, G. C., et al. 2012, ApJ, 747, 141

Hotan, A. W., van Straten, W., \& Manchester, R. N. 2004, PASA, 21, 302

Lazarus, P., Karuppusamy, R., Graikou, E., et al. 2020, CoastGuard: Automated Timing Data Reduction Pipeline, Astrophys. Source Code Libr., [record ascl:2003.008]

Lorimer, D. R., Bailes, M., McLaughlin, M. A., Narkevic, D. J., \& Crawford, F. 2007, Science, 318, 777

Maan, Y. 2015, ApJ, 815, 126

Maan, Y., \& Aswathappa, H. A. 2014, MNRAS, 445, 3221

Maan, Y., Deshpande, A. A., Chandrashekar, V., et al. 2013, ApJS, 204, 12

Maan, Y., Joshi, B. C., Surnis, M. P., Bagchi, M., \& Manoharan, P. K. 2019, ApJL, 882, L9

Nita, G. M., \& Gary, D. E. 2010, PASP, 122, 595

Nita, G. M., Gary, D. E., Liu, Z., Hurford, G. J., \& White, S. M. 2007, PASP, 119,805

Oostrum, L. C., van Leeuwen, J., Maan, Y., Coenen, T., \& Ishwara-Chandra, C. H. 2020, MNRAS, 492, 4825

Pastor-Marazuela, I., Connor, L., van Leeuwen, J., et al. 2020, ArXiv e-prints [arXiv:2012.08348]

Ransom, S. M., Eikenberry, S. S., \& Middleditch, J. 2002, AJ, 124, 1788

Sclocco, A., Vohl, D., \& van Nieuwpoort, R. V. 2019, 2019 RFI Workshop -

Coexisting with Radio Frequency Interference (RFI), [arXiv:2001.03389]

Smits, J. M., Mitra, D., \& Kuijpers, J. 2005, A\&A, 440, 683

Sosa Fiscella, V., del Palacio, S., Combi, L., et al. 2021, ApJ, 908, 158

Susobhanan, A., Maan, Y., Joshi, B. C., et al. 2021, PASA, 38, e017

Thornton, D., Stappers, B., Bailes, M., et al. 2013, Science, 341, 53

van Haarlem, M. P., Wise, M. W., Gunst, A. W., et al. 2013, A\&A, 556, A2

\footnotetext{
5 http://github.com/ymaan4/rficlean
} 